\title{
COLLABORATIVE EVENT MANAGEMENT IN SUPPLY CHAINS: AN AGENT-BASED APPROACH
}

\author{
Pascal Forget ${ }^{1,2}$ \\ Sophie D'Amours! \\ Jean-Marc Frayret' \\ ${ }^{\prime} F O R @$ C Research Consortium \\ Université Laval, Quebec, Canada \\ ${ }^{2} E$-mail: pascal.forget@centor:ulaval.ca
}

\begin{abstract}
The development of integrated supply chains and the use of interorganizational information systems have increased business interdependencies. Thus, the ability to deal quickly and seamlessly with everyday unplanned events is critical to maintain the overall performance of the supply chain. In order to develop tools to promote the collaborative management of such events, agent-based technology takes advantage of agents' ability to make autonomous decisions in a distributed context. Collaborative Event Management (CEM) is an approach designed to improve agility in a context where planning decisions are supported by a distributed advanced planning system (d-APS). This paper proposes an agent model geared with tools to collaboratively plan operations to deal with unplanned events.
\end{abstract}

\section{INTRODUCTION}

Events happen all the time. What is done is rarely done as planned. In manufacturing terms, we call these events, perturbations, disturbances, stochastic events, random events, uncertainties or contingencies. These events are in fact anything that moves production away from plan and requires planning adjustments. Machine breakdowns, sick leaves, supplier delays and planning errors are just a few examples of everyday events. The development of integrated supply chains and the use of inter-organizational information systems (IOIS) have increased business interdependencies. Managers know how to cope with events and solve the associated problems, but are they aware of the impacts of their decisions on partners? More precisely, are they taking into account the entire supply chain to make their decisions?

In fact, the majority of planning systems typically do not take events into account. The planning between different partners is done over a frozen horizon, with no disturbances, where variability is usually dealt with through inventory buffers and long lead times. If an event occurs, the plan is kept and actions are taken in the future to return as close as possible to the initial plan. As reaction time is generally 
slow, this method generates misleading decisions between the event and the actual response. On the other hand, centralized systems usually deal with events by replanning the whole plan or part of the whole plan, but in supply chains there are too many events and too many partners involved for re-planning at each change. Also, commercial supply chain event management systems usually only allows information about the occurrence of these events to be shared among supply chain partners, without any correction proposed. Distributed paradigms provide an interesting approach to permit local correction of the plan. In order to develop tools to promote the collaborative management of these events, agent-based technology provides a natural platform that takes advantage of the autonomy of agents and their ability to make decisions in a distributed context, using collaboration and goaldriven decisions following different events. An agent-based advanced planning and scheduling (APS) system could maintain a real-time plan by re-planning locally and allow for collaboration between agents to deal with events. Plan rectification in a short period of time leads the way to agile supply chains, fast reaction to events and increased global performance.

In this paper, we propose an agent-based model geared with tools to collaboratively plan production activities to deal with events in a distributed advanced planning system. We first provide a literature review on the use of agentbased technology in supply chains and existing agent-based collaborative schemes dealing with events. Next, we present the Collaborative Event Management (CEM) approach to improve the way events are handled in supply chains. Then, we introduce the agent-based experimental platform developed by the FOR@C Research Consortium, which is dedicated to supply chain planning for the forest industry. To profit from the advantages of CEM in a distributed environment such as the FOR@C platform, we detail a conceptual agent model showing suitable behaviors to deal with events in a distributed context. This takes advantage of the collaborative and goal-driven capabilities of agents. The North American lumber industry represents a perfect context for this technology. In fact, this industry is already highly distributed, where many business units interact in each production step. The main advantage of such technology in this industry is the large amount of stochastic events in many aspects of the supply chain, mainly due to the highly heterogeneous aspect of the resource, uncertain process output, production of coproducts and by-products, price variation on spot market and demand variation on commodity markets.

\section{LITERATURE REVIEW}

\subsection{Using agents in supply chain management}

Gathering information in a centralized management system and redistributing plans has been the standard for decades in supply chain planning systems. Although they were first elaborated to deal with a single enterprise, these information systems have now expanded to the entire supply chain. Generally speaking, they offer good results and represent an advantage for synchronizing the planning of many production units when coupled with decision support systems, such as APS. However, when the supply chain involves many partners planning problems become more complex. Also, because of quantities of information only locally available and the time it takes 
to plan the supply chain, plans are sometimes not feasible and the supply chain shows low reactivity. In fact, traditional systems have not been developed to work in decentralized, dynamic and heterogeneous environments (Maturana et al. 1999).

In recent years there is a new trend of management systems emerging, distributing decisions all over the supply chain that drifts away from traditional centralized management systems. Seen as networks of suppliers, factories, distribution centers and retailers, supply chains are increasingly complex, making it more difficult for management systems to stay agile and react quickly. This new trend resulted in the development of planning systems with agent-based architectures. Agents are defined as intelligent software, with specific roles and goals, interacting with each other to make the most appropriate decision according to the situation, in order to carry out their part of the planning task. Distributed planning shows many advantages over central planning. For complex problems, subproblems are easier to solve than centralized problems. Also, because decisions are distributed to different entities, reactivity to changes is increased and the feasibility of the plans is likely to be better. The challenge here is that plan performance is linked to agent collaboration capabilities to find good compromises. Agent-based technology has already been applied to different areas in supply chain management. For further details, the reader is referred to Parunak (1998) who presents industrial applications and case studies of agent-based systems, and Shen and Norrie (1999), who present descriptions of more than 30 research projects concerning on scheduling, planning and controlling. More recently, Caridi et al. (2004) present a survey and a classification of the different application domains of published multiagent projects, denoting their degree of maturity.

\subsection{Dealing with events}

Companies in the same supply chain have business interdependencies since the behavior of one can influence another. In a highly dependent network of entities, when activities are tightly planned, events can have important repercussions throughout the supply chain. For example, a major mechanical breakdown in a strategic third tier supplier can halt supply for several days, which will have tremendous impacts on the whole supply chain translating in a delay for the final client. Another example is a quick change in demand pattern. When such change happens, every demand plan exchanged between each partner must be updated. If it is not done in a very short period of time, stocks will pile-up and money will be wasted. This has increased in recent years as supply chain integration systems are adopted, CPFR (Collaborative Planning, Forecasting \& Replenishment) methodologies are followed and forecasts are prepared jointly. As stated in Frayret et al. (2004), interdependencies can vary greatly. The authors present a review of coordination structures, from centralized architecture to proper hierarchy, including heterarchy, to handle interdependencies. To permit a classification of events following their impact on the supply chain, Cloutier et al. (2001) present three levels of contingency management. These are local contingency management, local contingency management with need expression to partners and collaborative contingency management with eventual need expression to partners. Also, Davis (1993) presents a methodology developed with Hewlett-Packard for effectively addressing supply chain management, with interest on managing uncertainty in all supply chain processes. 


\subsection{Using agents to manage events}

Agent-based planning systems have been proposed to manage supply chains and deal with events. Montreuil et al. (2001) present the NetMan architecture, an operation system for networked manufacturing organizations geared up to cope with events not previously authorized. Although they created an architecture able to manage unseen events, they do not present specific behaviors to solve problems following perturbations. Based on intelligent holons, Fletcher et al. (2001) present a conceptual architecture of a lumber processing system to improve flexibility and fault tolerance. The ExPlanTech architecture developed by the Czech Technical University (Pechoucek et al. 2005) is a multiagent approach for decision-making support and simulation for manufacturing process. This approach integrates coordination and negotiation to deal with distributed environment. These architectures outline the possibility of reaction to however they do not propose solutions.

Conceptual agent models have also been proposed to improve supply chain performance and react to events. The Agent Building Shell (ABS) presented by Fox et al. (2000) is a collection of reusable software components and interfaces needed for any agent involved in a supply chain management system, geared to handle perturbations caused by stochastic events in a supply chain. In this architecture, most of the efforts have focused on defining communication and collaborative aspects. The InteRRaP agent model provides an interesting approach using a layered architecture able to react and deliberate when confronting changes, using different capability levels (Muller, 1996). This architecture is made of three layers, which are used to build action plans, depending if an event requires a reactive response, local planning or collaboration for planning. The tri-base acquaintance model (3bA) presented by Marik et al. (2005) is a collaborative wrapper added to an agent, giving the possibility to deal with events in a global perspective instead of resolving problems only in a local view. This is done by using information about other agents without the need of central facilitator. The authors present an example of applications in supply chains and they define Social knowledge needed to increase the efficiency of agents. From this review, we intend to propose a general approach to deal with event in a supply chain context.

\section{COLLABORATIVE EVENT MANAGEMENT}

Authors use different definitions when they discuss events. Fleury et al. (1999) present random events as anything implying delays in production time. The authors enumerate classes of reasons why such events occur: human mistake, failure of resource, failure of equipment and foreseeable production mistakes. Davis (1993) identifies three distinct sources of uncertainty, which are suppliers, manufacturing and customers. With the objective of mapping all the events encountered in daily production, we interviewed planners, directors and researchers involved in lumber supply chain planning activities. Inspired by Davis's methodology, we divided the events into three different levels, such as demand variation, execution variation and supply variation, and we noted all impacts on the supply chain. This investigation made clear that events have important impacts on production plans and ultimately, on financial results. To cope with events, lumber companies use inventory buffers or 
long lead times. While reducing event impacts, they increase inventory cost and have poor delivery lead times. This shows the importance of understanding events more precisely and how to react to them in an efficient way.

To emphasize the importance of collaboration when dealing with events in manufacturing systems in supply chains, we introduce Collaborative Event Management (CEM). This approach represents our conception on how collaboration should be exploited to deal with events within any manufacturing system in a supply chain, not limited to the lumber industry. Due to interdependencies between business partners, there is a need to coordinate the planning processes to solve problems resulting from events in a timely and efficient way. In a CEM perspective, we represent manufacturing activities in four different phases (see Figure 1), which are the Planning phase, the Scheduling phase, the Shop floor / Simulation phase and the Adjustment phase, representing the interactions between two different planning units. The first two phases are handled by the support staff and the two last are done by the operational staff.

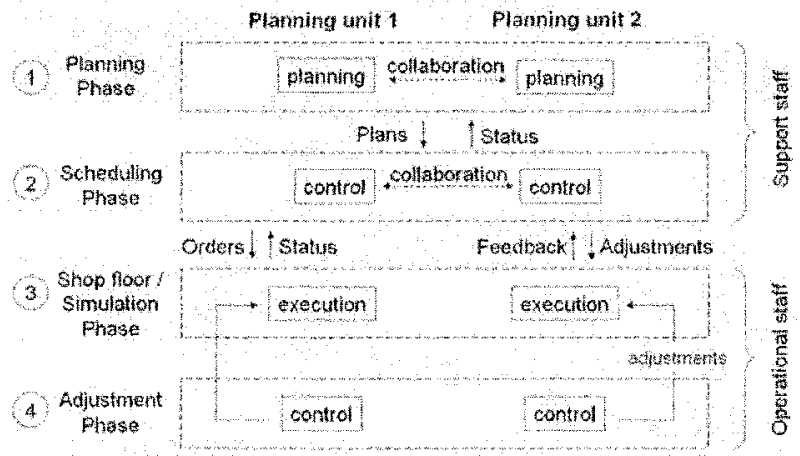

Figure 1 - Manufacturing phases in CEM

The Planning phase (1) includes the creation of the initial plan. From information exchanges between two different planners, they can collaborate and coordinate efforts for planning what product is needed and when. The Scheduling phase (2) dedicates resources to specific production tasks in a coordinated way. Status reports are redirected to the Planning phase, to make sure the initial plan is feasible with the resources available. The Shop floor / Simulation phase (3) is where the actual execution is done (or simulated). Orders from the Scheduling phase are transmitted and exact status is continually sent back to insure the plan is being followed. The Adjustment phase (4) works simultaneously with the third phase. It verifies resource status and monitors throughputs and reports to the Shop floor/Simulation phase for local adjustments. When adjustments are not possible because problems cannot be resolved at the Shop floor/Simulation phase without changing the plan, status reports are transmitted to higher levels. Because the last two phases are about local execution and control, there is no planning collaboration involved. Then, in the Scheduling or Planning phase, re-planning is executed, involving a single planning unit (locally) or many planning units (collaboratively).

CEM puts collaboration at the heart of the Planning and Scheduling phases. With extended collaboration protocols and anticipation of the impacts of their decisions, it is possible to propose problem solving techniques to face unforeseen contingencies. 
Such an approach can smooth transitions in the supply chain, reducing safety stocks and lead times usually kept to cope with undesired impacts. Applied to an agentbased planning platform, CEM provides input to create agents with appropriate characteristics.

\section{AGENT-BASED COLLABORATIVE EVENT MANAGEMENT}

\subsection{FOR@C experimental platform}

For many years, the planning processes in the North American lumber industry have never been questioned. Due to the highly heterogeneous nature of the resource (i.e. trees) and the inherent complexity of forecasting production throughput the dominant thinking was to produce the maximum volume with the resource available (push production). Because of the commodity nature of the final product and the standards of sizes and grades, to take advantage of economies of scale, production is oriented towards large batches. This industry can be characterized by large inventories, low flexibility and low agility. The recent economical and international threats to the lumber industry have encouraged some researchers to rethink the planning processes in a way to quickly react to correct deviance from the plan, respond to demand, reduce inventory and exchange information promptly throughout the supply chain (Frayret et al. 2005). In order to compensate for the lack of control over the stochastic elements relevant to lumber production, it is necessary to increase the exchange of information between the different production centers and to quickly react in a coordinated manner to changes.

With the purpose of developing a new planning approach for the lumber supply chain, the FOR@C Research Consortium of the Université Laval (Quebec, Canada) has developed an experimental planning platform built on an agent-based architecture for APS, with interaction mechanisms inspired from FIPA (Foundation for Intelligent Physical Agents) standards. This architecture combines agent technology with Operational Research (OR), in order to take advantage of the ability of agent-based technology to integrate distributed decision problems, and the ability of OR to solve complex decision problems (Frayret et al. 2005). Because of the distributed context of the supply chain and the use of agents, this platform can be described more precisely as a distributed-APS (or d-APS), where the first issue is to plan and coordinate all supply chain operations. As shown in Figure 2, the agentbased architecture presented by FOR@C is based on the natural division of the planning domains, where the production activities are divided among specialized agents: a sawing agent, a drying agent and a finishing agent. Each of these agents is responsible for supporting the planning of its production center in terms of production output each day. Other agents are also part of the architecture, such as the deliver agent, source agent and warehouse agent. Currently, testing is in progress with the collaboration of an international forest products company. The test uses 15 agents, more than 600 products, and approximately 80 exchange protocols, 100 tasks and 50 task flows. This architecture is a major step toward an improved coordination process for planning requirements. The main objective is to develop specific planning methods for each planning agents, using collaboration to insure synchronization in the supply chain. 


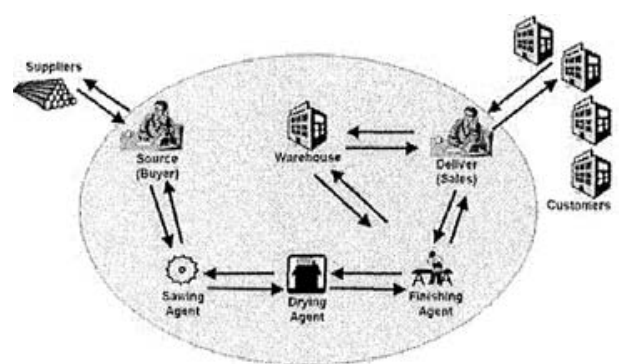

Figure 2 - Planning unit from the FOR@C platform

Each planning agent (such as sawing, drying and finishing agents) disposes of objects which are updated by local actions or actions from other agents. Actions are made possible by task flows, which are sequences of tasks, usually triggered by specific events. A standard task flow is the planning protocol (see Figure 3), triggered upon reception of a new demand plan from a client. This protocol is divided in two segments. The first is about modifying a requirement plan, creating a production plan with resource constraints and infinite supply, allocating demand to different suppliers and waiting for an answer. The second concerns receiving supply propositions, updating the production plan with a finite supply, allocating production to clients and modifying a replenishment plan. Optimization algorithms are deployed in the production planning (demand and supply propagation) and allocation tasks to suppliers and clients.

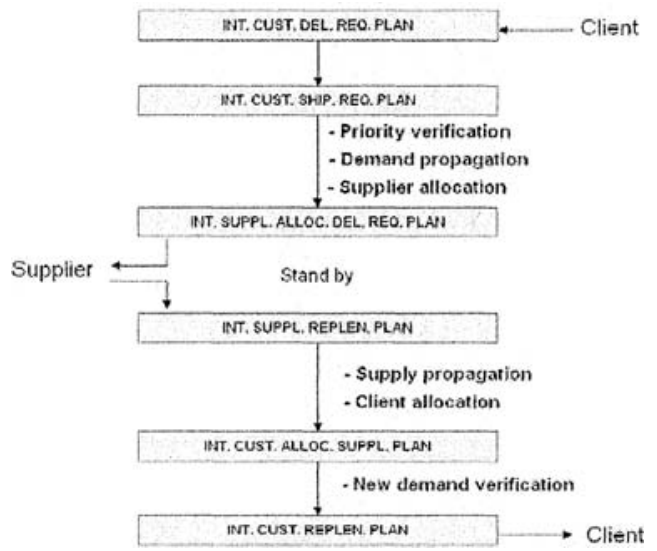

Figure 3 - Current planning protocol

\subsection{Enhancement of current planning agents}

Agent-based planning systems, such as the FOR@C experimental platform, represent a promising way to develop new planning systems and to apply the CEM approach. The next step is to develop an agent model to enhance the current planning agents of the experimental platform. Facing events, current agents use reactive task flows, triggered by specific messages. To deploy agents with behaviors 
adapted to different situations and environments, we must give the agent the possibility to make choices. Making clever choices requires sufficient knowledge and competencies to make good decisions. Also, the agent must understand the impacts of its decisions on itself, on other agents and on the whole supply chain.

\subsection{A conceptual agent model for CEM}

Our contribution in this paper is to describe a conceptual agent model that presents profitable behaviors to deal with events in a distributed collaborative context, using a CEM approach. Including similar concepts from previously presented models, we propose the Sense, Think \& Act (ST\&A) agent model (see Figure 4), integrating agent technology and OR tools. The model is composed of three distinct layers describing the different knowledge and competencies required to deal with events in supply chain planning. These layers can use different task flows, depending on their abilities to understand the impacts of their actions. In fact, layers represent what knowledge and competencies we wish to possess to face uncertain environment and be able to react efficiently to events. These layers do not depend on each other, since there is no strict passage from one to another for solving problems following events. In fact, it is an evolutive model, where the agent can have different intelligence levels in each layer, following its own evolution. Although presented as layers, the ST\&A agent is basically an evolutive conceptual model which the architecture is not necessarily layered, such as the InteRRaP architecture. The ST\&A conceptual model is similar, but its architecture can be completely different in its implementation.

ST\&A Agent

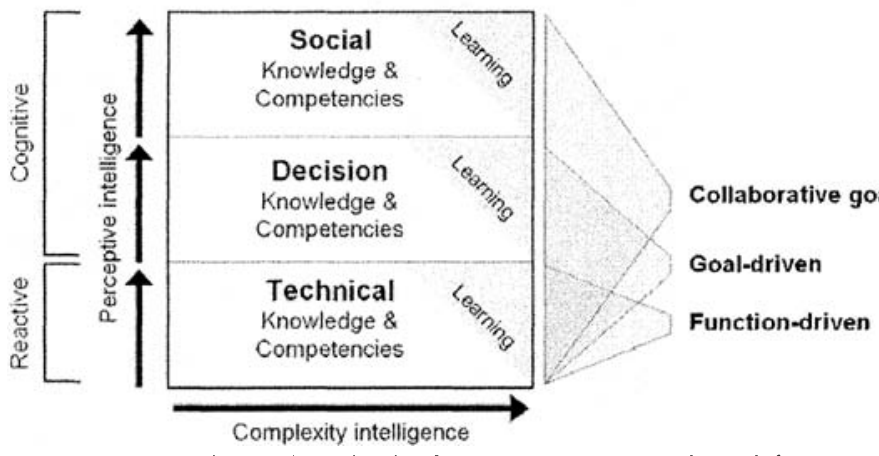

Figure 4 - The ST\&A agent conceptual model

The Technical layer is basically a reactive layer, which includes all reactive tools, tasks and existing task flows, such as OR tools and algorithms, conversation and negotiation protocols, and queries. Goals in this layer are related to minimizing effort (computer processing) while maximizing results (optimization functions included in tools). Reactive actions to face events are known and used when needed. An agent strong in this layer but weak in the others would show a function-driven behavior. The current agents deployed in the FOR@C platform present such behavior. Facing events, they send a new demand plan to suppliers and then, a new supply plan to clients. This is where a superior reasoning behavior would become interesting, giving new possibilities for dealing with disturbances, other than just start a global re-planning protocol. The Decision layer evolves from the reactive 
behavior to a cognitive behavior. It includes the explicit knowledge of local goals and the progress toward these goals at any time. These goals are towards local optimality, where the agent is concerned primarily with its own performance. This layer also encompasses the knowledge of the impacts of its decisions on itself. Here, when an event occurs, the agent has the capability to choose which task, task flow, optimization algorithms or complete plan could fit better, according to its own goals. An agent strong in its decision layer and technical layer would present a goal-driven behavior. The Social layer also denotes cognitive behavior, but here local goals are replaced by global goals. The agent is now aware of the impacts of its decisions on other agents and on the whole supply chain. While choosing actions to correct deviation from plan, we want the agent to possess the ability to capture the entire potential of the network and be able to minimize impact on others. Again, the agent has the capability to choose which task, task flow or plan responds the best to its own goals. It anticipates its environment (like other agent goals) and network tactical decisions (specific product, client selection, supplier selection, etc.) It has the ability to use collaboration protocols with anticipation of other agent reactions. Agents covering the three previous layers are considered collaborative goal-driven agents. Learning is a specific competency imbedded in each layer, which gives the agent the potential to increase its knowledge in each layer. A specific behavior that showed good results in a situation could be learned and remembered for next time. However, this subject will not be detailed in this article, but will be studied in details in close future.

With the objective to observe the behavior of the ST\&A agent in a CEM context, different event scenarios which confront planning agents have been developed, inspired from the lumber industry. This allows for comparing agent performances presenting different intelligence levels in each layer. Examples of scenarios are major dryer breakdown, out of stock and difference in harvest forecast. In a supplier/client relationship, we detailed the actions of these agents to solve the problem. The next step is to actually construct an agent architecture able to reproduce the behaviors denoted previously. Then, we will simulate different agent configurations by implementing them on the FOR@C agent-based platform.

\subsection{Toward a Collaborative Intelligence}

An agent showing collaborative behaviors, such as the collaboration-driven agent, uses Collaborative intelligence. Similar to the interpersonal intelligence in human intelligence (Gardner, 1983), we define Collaborative intelligence as the degree of knowledge and competencies toward using global goals to solve problems in planning activities. Indeed, this conceptual model represents what we consider to be required for a planning agent in a CEM context, as a complete collaborative goaldriven agent. Moreover, this model can represent a gradation tool to measure intelligence imbedded in any agent. In fact, each layer can be taken separately and scaled following degrees of achievement (as noted in Figure 4). We defined two axis of development; Complexity intelligence is the ability to deal with problem complexity and plurality of solutions, and Perceptive intelligence is defined as the degree of the environmental perception of the agent. This axis is scaled differently for each layer because achievements are different. Using this conceptual model, any planning agent could be positioned in the three layers and compared in its behavior confronting event management. 


\section{CONCLUSION}

Even if events are considered normal, they can still cause much trouble and costs (following Murphy's Law). The close management of events is an important opportunity to reduce inventory and lead times, at the same time increasing client satisfaction by delivering the right product at the right time. We believe collaboration is the key for handling events in the best way possible in a supply chain context. Approaches like CEM can be an interesting avenue to develop new collaboration protocols. Collaborative agent architecture using the advantage of $O R$, such as the ST\&A, can be a powerful tool to reach appreciated gains, when implemented in a distributed planning system. Following the conceptualization of the CEM approach and the ST\&A agent intelligence level descriptions, future work is needed. Implementation and testing of different agent configurations into the FOR@C experimental platform will be executed, in real-world planning situations. In a different perspective, it will be of a great interest to increase research efforts on the learning competency, on its implications and impacts. Already, research is on going and results will be available shortly.

\section{ACKNOWLEDGMENTS}

This work was funded by the FOR@C Research Consortium and the Natural Sciences and Engineering Research Council of Canada (NSERC).

\section{REFERENCES}

1. Caridi M, Cavalieri S. Multi-agent systems in production planning and control: an overview. Production Planning and Control, 15(2):106-118, 2004.

2. Cloutier L, Frayret JM, D'Amours S, Espinasse B, Montreuil B. A commitment-oriented framework for networked manufacturing co-ordination. International Journal of Computer Integrated Manufacturing, 14(6): 522-534, 2001.

3. Davis T. Effective Supply Chain Management. Sloan Management Review Summer 1993: 35-46.

4. Fox MS, Barbuceanu M, Teigen R. Agent-Oriented Supply-Chain Management. International Journal of Flexible Manufacturing Systems, 12:165-188, 2000.

5. Fletcher M, Brennan RW, Norrie DH, Fleetwood M. Reconfiguring processes in a holonic sawmill, 2001 IEEE International Conference on Systems, Man, and Cybernetics, 1:158-163, 2001

6. Frayret JM, D'Amours S, Rousseau A, Harvey S. Agent-Based Supply Chain Planning In The Forest Products Industry. Working Paper Dt-2005-Jmf-1, Centor, Université Laval, 2005.

7. Frayret JM., D'Amours S, Montreuil B. Coordination and Control in Distributed and Agent-Based Manufacturing Systems. Production Planning and Control, 15(1): 42-54, 2004.

8. Gardner G. Frames of mind: the theory of multiple intelligences. New York: Basic Books, 1983

9. Marik V, Pechoucek M, Stepankova O. Social Knowledge in Multi-agent Systems. Multi-Agent Systems and Applications, ACAI 2001, LNAI 2086, Springer, pp. 211-245, 2001

10. Maturana FP, Shen W, Norrie DH. Metamorph: An Adaptive Agent-Based Architecture for Intelligent Manufacturing. International Journal of Production Research, 37(10): 2159-2174, 1999.

11. Montreuil B, Frayret JM, D'amours S. A Strategic Framework for Networked Manufacturing. Computers in Industry, 42: 299-317, 2000.

12. Muller JP. The Design of Intelligent Agents: A Layered Approach. Lecture Notes in Artificial Intelligence, LNAI 1177, Springer, 1996.

13. Parunak, HVD. Practical and Industrial Applications of Agent-based Systems. Industrial Technology Institute, 1998.

14. Pechoucek M, Vokrinek J, Becvar P. ExPlanTech: Multiagent Support for Manufacturing Decision Making, IEEE Intelligent Systems, 20(1):67-74, 2005

15. Shen W, Norrie DH. Agent-Based Systems for Intelligent Manufacturing: A State-of-the-Art Survey. Knowledge and Information Systems, 1(2): 129-156, 1999. 